\title{
Avaliação de fragilidade, funcionalidade e medo de cair em idosos atendidos em um serviço ambulatorial de Geriatria e Gerontologia
}

\author{
Assessment of frailty, functionality and fear of falling in elderly assisted \\ at an outpatient gerontologic and geriatric clinic
}

\author{
Silvia Lanziotti Azevedo da Silva', Renata Alvarenga Vieira ${ }^{2}$, Paula Arantes ${ }^{3}$, Rosângela Corrêa Dias ${ }^{4}$
}

Estudo desenvolvido no Serviço Interdisciplinar de Geriatria e Gerontologia do Hospital Universitário da UFJF Universidade Federal de Juiz de Fora, Juiz de Fora, MG, Brasil

1 Fisioterapeuta; mestranda no PPG - Programa de Pós-

Graduação - em Ciências da Reabilitação da EEFFTO/

UFMG - Escola de Educação

Física, Fisioterapia e Terapia

Ocupacional da Universidade Federal de Minas Gerais, Belo Horizonte, MG

2 Doutoranda no PPG em Ciências da Reabilitação da EEFFTO/UFMG; Profa. Ms. do Depto. de Fisioterapia da Faculdade de Medicina da UFJF

3 Fisioterapeuta; doutoranda no PPG em Ciências da Reabilitação da EEFFTO/UFMG

4 Profa. Dra. Associada do Depto. de Fisioterapia da EEFFTO/UFMG

\section{ENDEREÇO PARA} CORRESPONDÊNCIA

Silvia L. A. da Silva R. João Antônio Cardoso 157 ap. 301

31310-340 Belo Horizonte MG e-mail: silviafisiojf@yahoo.com.br

APRESENTAÇÃO out. 2008

ACEITO PARA PUBLICAÇÃO maio 2009
Resumo: A síndrome da fragilidade é uma condição associada ao envelhecimento, com desfechos de saúde nos idosos como quedas, medo de cair e incapacidade. Os objetivos deste estudo foram determinar a freqüência de fragilidade e verificar a existência de correlação desta com quedas, medo de cair e funcionalidade, em 30 idosos (média de 75,7 7,6 anos) cadastrados em um serviço interdisciplinar de Geriatria e Gerontologia. Foram avaliados quanto à fragilidade, por características sugeridas pela literatura, e quanto aos desfechos quedas, funcionalidade e medo de cair. A análise descritiva identificou $20 \%$ de idosos frágeis, $46,7 \%$ pré-frágeis e $33,3 \%$ não-frágeis. Foram encontradas diferenças significativas entre os grupos frágeis e pré-frágeis e frágeis e não-frágeis em relação à funcionalidade mensurada pela escala de Lawton $(p=0,000)$ e medo de cair, avaliada pela escala internacional de eficácia de quedas (FES-I, na sigla em inglês). Foi encontrada correlação significativa e moderada entre a pontuação na FES-I e o número de quedas; e correlação significativa, moderada e inversa, entre as pontuações da FES-I e de Lawton. A freqüência de fragilidade foi maior na amostra do estudo do que a encontrada em estudos prévios; e foram encontradas diferenças significativas entre os grupos de idosos, de acordo com as características de fragilidade, indicando que os mais frágeis apresentavam maior incapacidade para atividades de vida diária e mais medo de cair.

Descritores: Avaliação geriátrica; Idoso fragilizado

ABSTRACT: The frailty syndrome is a condition associated to age-related vulnerability, bearing health outcomes such as falls, fear of falling, and disability. The purposes of this study were to determine frequency of frailty and to search for correlations between frailty and falls, fear of falling, and functionality, in a group of 30 elderly (mean age $75.7 \pm 7.6$ ) registered at a geriatric outpatient clinic. They were assessed as to frailty according to features suggested by literature, and as to the outcomes falls, fear of falling, and functionality. The sample descriptive analysis identified $20 \%$ of frail, $46.7 \%$ of pre-frail and $33.3 \%$ of non-frail elderly. Significant differences were found between frail and pre-frail, and between frail and non-frail groups, as to functionality as measured by the Lawton scale $(p=0.000)$, and as to fear of falling (assessed by the Falls Efficacy Scale - FES-I). A moderate, significant correlation was found between FES-I scores and the number of falls; and a moderate, inverse, significant correlation between FES-I scores and Lawton scale scores. Frailty frequency in the present study was greater than in previous ones. The significant differences found between groups according to frailty classification suggest that the most frail subjects had greater incapacity for activities of daily living and more fear of falling.

KeY words: Frail elderly; Geriatric assessment 


\section{INTRODUÇÃO}

O crescimento da população idosa é um fenômeno mundial e, no Brasil, as modificações acontecem de forma acelerada e significativa ${ }^{1}$. Acredita-se que o país será, em 2025, o sexto do mundo em número absoluto de idosos, totalizando 33,8 milhões de indivíduos nessa faixa etária, com sua proporção evoluindo de $2,7 \%$ para $14,7 \%$ da população ${ }^{2}$. Diante desse envelhecimento populacional, novas demandas de saúde emergem.

Várias condições se associam à saúde dos idosos, dentre as quais a síndrome da fragilidade. Essa síndrome é uma condição multissistêmica, mais prevalente com o aumento da idade ${ }^{3}$, definida por Fried et al. 4 como estado de vulnerabilidade crescente a estressores, resultado do declínio das reservas fisiológicas e desregulação de múltiplos sistemas, limitando a capacidade de manter a homeostase diante de estressores. Buscando identificar a síndrome da fragilidade em idosos, Fried et al. ${ }^{5}$ reuniram condições como emagrecimento ligado à desnutrição e perda de massa muscular, fraqueza muscular, baixa resistência, lentidão e baixos níveis de atividade, para formular um fenótipo de fragilidade. Tal fenótipo foi definido pelo autorrelato de exaustão e perda involuntária de peso no último ano, perfil de atividade física, força de preensão manual e velocidade de marcha. Segundo essas características, os idosos são classificados como frágeis, quando apresentam três ou mais dos componentes citados, pré-frágeis, quando apresentam um ou dois deles, e não-frágeis, quando não apresentam qualquer dessas características $^{6}$. Foi demonstrado que esse fenótipo apresenta validade interna e de critério e é capaz de predizer vários desfechos clínicos, como quedas, incapacidades, hospitalização e morte ${ }^{5}$.

Embora não seja sinônimo de tais condições, a fragilidade é relacionada à presença de comorbidades e incapacidade, podendo ser causada ou predispor tais eventos ${ }^{4}$. Um estudo longitudinal $^{7}$ com mulheres idosas apontou que $56 \%$ daquelas consideradas frágeis, em um primeiro inquérito, desenvolveram alguma incapacidade para pelo menos uma atividade básica de vida diária (ABVD) durante o acompanhamento, enquanto $20 \%$ das consideradas não-frágeis se tornaram dependentes. Frente ao caráter negativo e prejudicial de tais desfechos, torna-se importante identificar relações entre eles e a síndrome da fragilidade em amostras variadas a fim de orientar a atenção à saúde dos idosos, visando a tomada de decisões e o planejamento de ações de educação, prevenção e promoção da saúde. Assim, quanto maior o número de estudos em diferentes populações de idosos, maior o número de evidencias disponíveis para os profissionais de saúde na assistência à síndrome, justificando estudos como este.

O objetivo deste estudo foi avaliar a freqüência de fragilidade em um grupo de idosos atendidos em um serviço interdisciplinar de Geriatria e Gerontologia e sua relação com quedas, funcionalidade e medo de cair.

\section{METODOLOGIA}

O projeto deste estudo foi submetido à avaliação do Comitê de Ética em Pesquisa da UFJF - Universidade Federal de Juiz de Fora. Antes do início da entrevista todos os sujeitos assinaram um termo de consentimento livre e esclarecido. Para identificação do fenótipo de fragilidade e seus fatores associados foi realizado um estudo clínico observacional com delineamento transversal.

A população do estudo foi composta por idosos cadastrados no Serviço Interdisciplinar de Geriatria e Gerontologia do Hospital Universitário da UFJF. No momento do levantamento o serviço assistia 63 idosos. Os critérios de inclusão no estudo foram idade superior a 60 anos e ser cadastrado no Serviço; os critérios de exclusão foram apresentar deficit cognitivo, definido de acordo com a pontuação no teste do mini-exame do estado mental (MEEM) descrito por Bertolucci et al. ${ }^{8}$, e estar acamado ou ser cadeirante.

Pela análise das fichas de avaliação dos pacientes em sua primeira consulta, foram excluídos cinco idosos que haviam falecido e cinco com alteração cognitiva grave ou demência registrados desde a entrada no Serviço, restando 53 idosos para compor a amostra. Em seguida foi feito contato telefônico com o paciente e convite para participação no estudo. O contato com seis pacientes não foi possível devido a falhas na notificação no prontuário. Assim, foram contatados 47 pacientes, dentre os quais dois foram excluídos por terem desenvolvido alterações cognitivas que impossibilitavam a participação, três haviam falecido sem notificação ao Serviço, um tinha idade inferior a 60 anos e três não residiam no município, não podendo comparecer ao local de coleta de dados. Nove idosos recusaram-se a participar do estudo. Por fim, 30 idosos compuseram a amostra estudada.

\section{Procedimentos}

A coleta de dados foi realizada em consultório no Hospital onde funciona o Serviço ao qual os pacientes eram vinculados. Eles aí compareceram mediante agendamento de horário, após terem aceitado participar.

Inicialmente foi aplicado o MEEM, para rastreamento de alterações cognitivas. De acordo com o nível de escolaridade, os idosos deveriam pontuar no mínimo 24, 18 e 13 pontos para alta, média, baixa escolaridade e analfabetos, respectivamente ${ }^{8}$. Todos alcançaram a pontuação mínima e prosseguiram na avaliação.

Foram coletados os dados que, segundo a literatura, poderiam estar relacionados à presença de fragilidade, como variáveis sociodemográficas, saúde física percebida (por autoavaliação), diagnóstico médico de doenças prevalentes em idosos e número de medicamentos utilizados. O desempenho nas atividades instrumentais de vida diária (AIVD), como uso de medicação, preparo de refeições, manejo do dinheiro, foi avaliado pela escala de Lawton ${ }^{9}$, onde os escores variam de 7 a 21 e, quanto maior o escore, melhor o desempenho; e as atividades básicas de vida diária (ABVD, ligadas ao autocuidado, higiene, alimentação), pela escala de Katz (versão brasileira $\left.{ }^{10}\right)$; nesta os escores vão de 0 a 6 e, quanto mais alto o escore, pior o desempenho. 
Os idosos também foram inquiridos sobre medo de cair e quedas. Estas foram investigadas por um questionário estruturado, iniciado pela pergunta, "O/ A senhor/a sofreu queda no último ano?". O medo de cair foi avaliado pela escala de eficácia de quedas FES-I (Falls efficacy scale-International) ${ }^{11}$, com escore máximo de 64 pontos, sendo que escores inferiores a 23 pontos apontam para maior independência - ou seja, a pontuação mais elevada indica mais medo de sofrer uma queda.

A presença de fragilidade foi verificada por testes para identificação dos cinco itens descritos como componentes do fenótipo de fragilidade por Fried et al. ${ }^{5}$ - perda de peso, exaustão, nível de atividade física, lentidão na marcha e fraqueza muscular - da forma proposta por esses autores. A perda de peso é considerada por autorrelato de perda superior a 4 kg no último ano; exaustão é avaliada pela concordância "sempre" ou "na maioria das vezes" a duas afirmativas - "Senti que tive de fazer esforço para fazer tarefas habituais" e "Não consegui levar adiante minhas coisas" (que integram uma escala de avaliação da depressão da instituição norte-americana de estudos epidemiológicos, CES-D). O nível de atividade física foi aferido pela versão curta do questionário de atividades de lazer de Minnesota (Minnesota leisure time activity questionnaire), com perguntas sobre hábitos de andar, correr, jardinagem, entre outras, cuja mensuração de gasto calórico é feita por algoritmos; a lentidão da marcha foi medida pelo tempo gasto para percorrer a distância de 4,6 m, em velocidade confortável, ajustada por sexo e altura; por fim, a força muscular foi verificada com base na força de preensão mensurada em Kgf por dinamômetro de preensão manual (Jamar), sendo o resultado ajustado por sexo e índice de massa corporal (IMC).

Idosos que tivessem resultados positivos em três ou mais dos cinco itens avaliados para identificação do fenótipo são considerados frágeis; aqueles com um ou dois resultados positivos, pré-frágeis; e aqueles com todos os testes negativos para fragilidade são considerados robustos ou não-frágeis. Os pontos de corte adotados pelo estudo para classificação dos itens velocidade de marcha e força de preensão manual para o sexo feminino foram os estabelecidos para idosos brasileiros por Santos et al. ${ }^{12}$.

\section{Análise estatística}

Os dados foram analisados descritivamente por meio do pacote estatístico SPSS 13.0. Uma análise de variância (Anova unidirecional) foi utilizada para comparar os escores das escalas FES-I, Lawton e Katz-Brasil entre os grupos frágil, pré-frágil e não-frágil. O coeficiente de correlação de Spearman foi utilizado para avaliar a correlação entre estas variáveis e o número de quedas sofridas. Para a comparação do número de idosos com história de quedas em cada grupo foi utilizado o teste exato de Fisher. Todas as análises foram consideradas no nível de significância $p<0,05$.

\section{RESULTADOS}

A população do estudo foi constituí- da por 10 homens e 20 mulheres, com média de idade de $75,7 \pm 7,6$ anos, e média do índice de massa corporal (IMC) de $23,9 \pm 3,8$. A maioria eram casadas/os (40,0\%) ou viúvas/os (11/36,7\%), brancas/ os (70\%); $90 \%$ não trabalhavam atualmente, sendo 19 (quase dois terços) aposentados. A maioria (40\%) tinham apenas até 4 anos e $23 \%$ eram analfabetos. Os idosos tinham tido uma média de 2,9 filhos e 73,3\% deles não moravam sozinhos.

A maioria dos idosos apresentavam comorbidades como hipertensão, alterações cardiovasculares, diabetes, alterações visuais, alterações reumáticas, entre outras, e usavam medicamentos. As características clínicas e funcionais da amostra são apresentadas na Tabela 1.

Como mencionado, para efeito de análise os idosos foram reunidos em três grupos, de frágeis $(n=6)$, pré-frágeis $(n=14)$ e não-frágeis $(n=10)$. Não foi encontrada diferença estatisticamente significativa em relação ao número de ido-

Tabela 1 Características clínicas e funcionais da amostra $(n=30)$

\begin{tabular}{|c|c|c|c|}
\hline Característica & & $\mathrm{n}$ & $\%$ \\
\hline Hipertensão arterial sistêmica & Sim & 17 & 6,7 \\
\hline & Não & 13 & 43,3 \\
\hline Alterações visuais & Sim & 26 & 86,7 \\
\hline & Não & 4 & 13,3 \\
\hline $\mathrm{N}$ comorbidades por idoso (me & dia \pm dp) & $3,5 \pm 1,7$ & \\
\hline Uso de medicação & Sim & 24 & 80,0 \\
\hline & Não & 6 & 20,0 \\
\hline Consumo de medicação & $>$ quatro & 12 & 50,0 \\
\hline & $<$ quatro & 12 & 50,0 \\
\hline Autoavaliação de saúde & Muito boa & 1 & 3,3 \\
\hline & Boa & 16 & 53,5 \\
\hline & Regular & 12 & 40,0 \\
\hline & Ruim & 1 & 3,3 \\
\hline Independência em AIVD & Independente & 22 & 73,3 \\
\hline 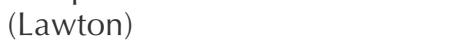 & Realiza com ajuda & 5 & 16,7 \\
\hline & Dependente & 3 & 10,0 \\
\hline Independência em ABVD (Katz) & em 6 funções & 23 & 76,7 \\
\hline Dependente & em pelo menos uma função & 7 & 23,3 \\
\hline Quedas no último ano & Sim & 20 & 66,7 \\
\hline & Não & 10 & 33,3 \\
\hline Conseqüências da queda & Dor & 7 & 35,0 \\
\hline & Escoriação/corte/contusão & 5 & 25,0 \\
\hline & Fratura & 0 & 0,0 \\
\hline Caiu ao realizar & AIVD & 11 & 55,0 \\
\hline & ABVD & 9 & 45,0 \\
\hline
\end{tabular}

$\mathrm{dp}=$ desvio padrão; AIVD= atividades instrumentais de vida diária; ABVD = atividades básicas de vida diária; FES-I= Falls Efficacy Scale International 
Tabela 2 Escores (média \pm dp) nas escalas de Lawton (desempenho nas AIVD) e FES-I (medo de queda) dos grupos de idosos frágeis (Fr), pré-frágeis (PF) e não-frágeis (NF) e comparação entre os grupos $(n=30)$

\begin{tabular}{lccc}
\hline $\begin{array}{l}\text { Escores nas } \\
\text { escalas }\end{array}$ & Frágeis $(\mathrm{n}=6)$ & $\begin{array}{c}\text { Grupos de idosos } \\
\text { Pré-frágeis }(\mathrm{n}=14)\end{array}$ & Não-frágeis $(\mathrm{n}=10)$ \\
\hline AIVD (Lawton) & $14,33 \pm 4,5$ & $19,57 \pm 2,0$ & $20,6 \pm 0,9$ \\
$p$ da diferença & Fr vs NF $p=0,000^{*}$ & Fr vs PF $p=0,000^{*}$ & NF vs PF $p=0,394$ \\
FES-I & $33,3 \pm 7,3$ & $24,9 \pm 4,2$ & $20,6 \pm 0,9$ \\
$p$ da diferença & Fr vs NF $p=0,039^{*}$ & Fr vs PF $p=0.011^{*}$ & NF vs PF $p=0,748$ \\
\hline
\end{tabular}

$\mathrm{dp}=$ desvio padrão; AIVD = atividades instrumentais da vida diária; FES-I = Falls Efficacy Scale International; * estatisticamente significante $(p<0,05)$

sos com história de quedas em cada grupo. Em relação ao sexo e idade, também não foram encontradas diferenças significativas entre os grupos.

Quanto à pontuação na escala de Lawton, que avalia a independência em atividades instrumentais da vida diária, os idosos frágeis tiveram, como era de se esperar, escores mais baixos, com diferença estatisticamente significativa entre eles e os não-frágeis, e entre eles e os pré-frágeis (Tabela 2). A pontuação na FES-I, que avalia o medo de quedas, também mostrou que os idosos frágeis tiveram escores mais altos, com diferença estatisticamente significativa entre os idosos frágeis e não-frágeis e entre frágeis e pré-frágeis (Tabela 2).

Independentemente dos grupos, foi encontrada correlação significativa e moderada entre a pontuação na FES-I e o número de quedas no ano anterior $(r=0,503 ; p=0,005)$ e correlação significativa, moderada e inversa entre os escores na FES-I e na escala de Lawton $(r=0,565 ; p=0,001)$.

Tabela 3 Correlações entre número prévio de quedas e os escores médios nas escalas de Lawton e FES-I em toda a amostra $(n=30)$

\begin{tabular}{lccc}
\hline & FES-I & Lawton & $\mathrm{N}$ quedas \\
\hline Lawton & $-0,565^{*}$ & 1,000 & $-0,332$ \\
N quedas & $0,503^{*}$ & $-0,332$ & 1,000 \\
FES-I & 1,000 & $-0,565^{*}$ & $0,503^{*}$
\end{tabular}

* Correlação significante $p<0,01$

\section{DISCUSSÃO}

A freqüência de fragilidade encontrada na amostra (20\%) foi mais elevada do que a reportada pela literatura ${ }^{5,6,13}$.
O pequeno tamanho da amostra deste estudo e uma maior complexidade do estado de saúde dos pacientes cadastrados no Serviço, situado dentro de um hospital, poderiam justificar tal diferença. Santos et al. ${ }^{12}$ trabalharam com uma amostra de conveniência de 113 idosos da comunidade assistidos em unidades básicas de saúde e de um centro de referência de idosos de um hospital universitário, a fim de verificar a prevalência de fragilidade. Encontraram 10,6\% de idosos frágeis, $43,4 \%$ de pré-frágeis e $43,4 \%$ de não-frágeis, após ajuste dos pontos de corte das variáveis do fenótipo na amostra. Foram adotados, no presente estudo, os mesmos pontos de corte daqueles autores pois, apesar de esta amostra ser menor, os idosos eram também vinculados a serviços secundários de saúde e amostrados por conveniência.

No presente estudo, a análise descritiva da amostra divida por perfis de fragilidade revelou maior percentual de idosos viúvos e que vivem sozinhos entre aqueles classificados como não-frágeis. Segundo Woods et al. ${ }^{13}$, o idoso mais fragilizado perde a independência e a capacidade de morar sozinho. Isso é corroborado pelo fato de que os idosos não-frágeis da amostra do presente estudo mostraram-se mais independentes nas AIVD e ABVD, o que também ocorreu no estudo de Santos et al. ${ }^{12}$.

As patologias mais comuns observadas entre os idosos da amostra foram hipertensão arterial e alterações visuais. O estudo de Brandão et al. ${ }^{14}$ observou aumento da pressão arterial em $60 \%$ dos idosos estudados. Nesta pesquisa tal condição apareceu em 56,7\% da amostra. Alterações visuais foram encontradas em $86,7 \%$ da amostra, semelhante ao encontrado por Sampaio e Boaret ${ }^{15}$, que relataram $90 \%$ de alterações da vi- são em idosos. Contudo, não foram encontradas diferenças estatisticamente significativas dessas variáveis entre os grupos de idosos frágeis, pré-frágeis e não-frágeis, devido provavelmente ao tamanho reduzido da amostra e diferenças no número de idosos em cada grupo.

Ainda em relação às comorbidades, a análise neste estudo revelou que os idosos não-frágeis apresentavam maior média de doenças acumuladas que os pré-frágeis, o que está de acordo com a evidência de que fragilidade e comorbidade são conceitos distintos e podem existir independentemente ${ }^{4}$. Wilson ${ }^{16}$ mostrou que, mesmo quando indivíduos com condições agudas e crônicas eram excluídos da análise, $7 \%$ da população com mais de 65 anos era considerada frágil; e, quando analisados os idosos com mais de 80 anos, tal percentual subia para $20 \%$. Também no estudo de Santos et al. ${ }^{12}$, os idosos frágeis tiveram quase duas vezes mais chances de acumular comorbidades, mas 6,3\% deles não apresentavam doença alguma e $6,3 \%$, apenas uma.

O presente estudo mostra que os idosos pré-frágeis obtiveram média na escala de Lawton inferior à dos não-frágeis e superior à dos frágeis, com diferenças significativas. Isso está de acordo com o reportado por Topinková ${ }^{17}$, de que a relação entre incapacidade e fragilidade é evidenciada nas AIVD. Neste estudo, 33,3\% dos idosos frágeis necessitavam de alguma ajuda e $50 \%$ eram dependentes de outras pessoas para realização das AIVD. Já em relação às $A B V D$, não foram encontradas diferenças estatisticamente significativas entre o desempenho nos três grupos de idosos. Na linha de base do estudo de Woods et al. ${ }^{13}$, dependência para pelo menos uma ABVD esteve mais presente no grupo de idosas consideradas frágeis do que nas não-frágeis. As idosas que se tornaram frágeis durante o acompanhamento longitudinal foram as que relataram maior dependência em relação à ABVD.

Apesar de a diferença não ter sido significativa, a análise descritiva mostra que a proporção de idosas que perderam pontos na escala de Katz-Brasil foi maior entre as idosas não-frágeis do que entre as frágeis. Uma possível explicação para tal fato é que a escala de Katz questiona 
sobre a continência em um de seus domínios ${ }^{10}$, e algumas idosas não frágeis da amostra relataram incontinência urinária, e, por ser uma amostra pequena, este fato pode ter gerado essa diferença observada na análise descritiva.

A diferença na pontuação da FES-I foi estatisticamente significativa entre os grupos de idosos frágeis e pré-frágeis, e entre frágeis e não-frágeis, indicando maior medo de cair naqueles classificados como frágeis. Arfken et al. ${ }^{18}$ definiram de forma diferente a fragilidade, mas ainda assim acharam correlação significativa entre fragilidade e medo de quedas. Kressig et al. ${ }^{20}$, usando o conceito de fragilidade de Tinetti e Speechely ${ }^{19}$, encontraram relação entre presença de fragilidade e medo de cair.

Não foram encontradas diferenças significativas entre os três grupos em relação à ocorrência ou não de quedas no ano anterior à aplicação do questionário, apesar de a literatura relatar que idosos frágeis têm maiores chances de sofrer quedas ${ }^{5}$. Isso poderia ser explicado pelo fato de as quedas relatadas por $66,7 \%$ dos idosos da amostra do presente estudo terem ocorrido, em sua maioria, enquanto realizavam atividades mais complexas (e não em atividades da vida diária), ex- pondo assim aqueles não-frágeis e mais independentes a riscos nessas atividades.

Correlações positivas foram encontradas entre desempenho na escalas de Lawton e FES-I, assim como entre desempenho na FES-I e número de quedas no ano anterior. Tais correlações foram observadas na análise da amostra como um todo, sem divisão entre grupos de acordo com fragilidade. A correlação significativa entre FES-I e quedas está de acordo com a evidência de que quanto maior o medo de quedas no idoso, maior o risco que ele tem de sofrer tal evento ${ }^{21}$.

A relação inversa entre a FES-I e a de Lawton ocorre devido à pontuação nas duas escalas. A escala de Lawton considera mais independente aquele idoso que alcança maior pontuação ${ }^{9}$, enquanto a FES-I considera assim aquele que tem menos pontos acumulados ao longo da escala ${ }^{11}$. No presente estudo, os idosos mais independentes e com maior pontuação acumulada ao longo da escala de Lawton tiveram menos pontos na FES-I. Cumming et al. ${ }^{22}$ encontraram correlação positiva entre o medo de cair avaliado pela FES-I e dependência em atividades de vida diária, apesar de estas não terem sido operacionalizadas pela escala de Lawton.

\section{CONCLUSÃO}

Apesar de este estudo ter avaliado uma amostra peculiar de um serviço e utilizado instrumentação que restringia essa amostra àqueles idosos com bom nível cognitivo, não-acamados e não-cadeirantes, foi possível traçar o perfil dos mesmos, tendo-se encontrado diferenças significativas entre os grupos de frágeis e pré-frágeis e entre frágeis e não-frágeis. A proporção de fragilidade foi maior na amostra deste estudo do que a encontrada em estudos prévios e houve diferenças significativas entre os grupos de idosos de acordo com as características de fragilidade, indicando que os mais frágeis apresentavam maior incapacidade para atividades da vida diária e mais medo de cair.

Com o crescimento dos estudos acerca desta temática, torna-se cada vez mais importante identificar idosos frágeis e pré-frágeis nos vários níveis de atenção à saúde, permitindo a adequação dos serviços às novas demandas relacionadas ao envelhecimento. Espera-se, então, que os dados obtidos venham a possibilitar melhores tomadas de decisão clínica em relação à prevenção e intervenção terapêutica em serviços de saúde similares, assim como acumular informações sobre a síndrome da fragilidade.

\section{REFERÊNCIAS}

1 Veras R. Envelhecimento populacional e as informações de saúde do PNAD: demandas e desafios contemporâneos. Cad Saude Publica. 2007;23:2463-66.

2 Veras R. Considerações acerca de um país jovem que envelhece. Cad Saude Publica. 1988;4:382-97.

3 Toniolo Neto J, Ramos LR. Guia de Geriatria e Gerontologia. São Paulo: Manole; 2005.

4 Fried LP, Ferrucci L, Darer J, Williamson JD, Anderson G. Untangling the concepts of disability, frailty, and comorbidity: implications for improved targeting and care. J Gerontol A Biol Sci Med Sci. 2004;59:255-63.

5 Fried LP, Tangen CM, Walston J. Frailty in older adults: evidence of a phenotype. J Gerontol A Biol Sci Med Sci. 2001;56A:146-56.

6 Bandeen-Roche K, Xue QL, Ferrucci L, Walston J, Guralnik JM, Chaves P, et al. Phenotype of frailty: characterization in the women's health and aging studies. J Gerontol A Biol Sci Med Sci. 2006;6:262-6.
7 Boyd CM, Xue QL, Simpson CF, Guralnik JM, Fried LP. Frailty, hospitalization, and progression of disability in a cohort of disabled older women. Am J Med. 2005;118:1225-31.

8 Bertolucci PHF. O mini-exame do estado mental em uma população geral: impacto da escolaridade. Arq Neuropsiquiatr. 1994;52:1-7.

9 Lawton MP, Brody EM. Assessment of older people: selfmaintaning and instrumental activities of daily living. Gerontologist. 1969;9:179-86.

10 Lino VTS, Pereira SRM, Camacho LAB, Ribeiro Filho ST, Buksman S. Adaptação transcultural da escala de independência em atividades da vida diária (Escala de Katz). Cad Saude Publica. 2008;24:103-12.

11 Camargos F. Adaptação transcultural e avaliação das propriedades psicométricas da Falls Efficacy Scale International: um instrumento para avaliar medo de cair em idosos [dissertação] Belo Horizonte: Programa de Pós-Graduação em Ciência da Reabilitação da Escola de Educação Física, Fisioterapia e Terapia Ocupacional, Universidade Federal de Minas Gerais; 2007. 
12 Santos EGS. Perfil de fragilidade em idosos comunitários de Belo Horizone: um estudo transversal [dissertação]. Belo Horizonte: Escola de Educação Física, Fisioterapia e Terapia Ocupacional da Universidade Federal de Minas Gerais; 2008.

13 Woods NF, LaCroix AZ, Gray SL, Aragary A, Cochrane $\mathrm{BB}$, et al. Frailty: emergence and consequences in women aged 65 and older in the Women's Health Initiative observational study. J Am Geriatr Soc. 2005;53:1321-30.

14 Brandão AP, Brandão AA, Magalhães MEC, Pozzan R Epidemiologia da hipertensão arterial. Rev Soc Cardiol. 2003;13:7-19.

15 Sampaio LFR, Boaretto MC. Cadernos de atenção básica 19: envelhecimento e saúde da pessoa idosa. Brasília: Ministério da Saúde; 2006.

16 Wilson JF. Frailty - and its dangerous effects - might be preventable. Ann Intern Med. 2004;141:489-92.

17 Topinková E. Aging, disability and frailty. Ann Nutr Metab. 2008;52:6-11.
18 Arfken CL, Lach HW, Birge SJ, Miller P. The prevalence and correlates of fear of falling in elderly persons living in the community. Am J Public Health. 1994;84:565-70.

19 Speechely M, Tinetti M. Falls and injuries in frail and vigorous community elderly persons. J Am Geriatr Soc. 1991;39:46-52.

20 Kressig RW, Wolf SL, Sattin RW, O'Grady M, Greenspan A, Curns A, et al. Associations of demographic, functional, and behavioral characteristics with activityrelated fear of falling among older adults transitioning to frailty. J Am Geriatr Soc. 2001;49:1456-62.

21 Zijstra GAR, Van Haastregt JCM, Van Rossun E, Van Ejik JTM, Kempen GIJM. Interventions to reduce fear of falling in community-living older people: a systematic review. J Am Geriatr Soc. 2007;55:603-15.

22 Cumming RG, Salkeld G, Thomas M, Szonyi G. Prospective study of the impact of fear of falling on activities of daily living, SF-36 scores, and nursing home admission. J Gerontol A Biol Sci Med Sci. 2000;55:299-305. 